(2) Open Access Full Text Article

ORIGINAL RESEARCH

\title{
Curcumin alleviates rheumatoid arthritis-induced inflammation and synovial hyperplasia by targeting mTOR pathway in rats
}

This article was published in the following Dove Press journal:

Drug Design, Development and Therapy

\section{Qiaoding Dai' \\ Di Zhou ${ }^{2}$ \\ Liping $X u^{1}$ \\ Xinwei Song'}

'Department of Rheumatology and Immunology, First Affiliated Hospital of Zhejiang Chinese Medical University, Hangzhou 310006, Zhejiang, People's Republic of China; ${ }^{2}$ Nephrology Department, First People's Hospital of Xiaoshan District, Hangzhou 3 I I 200, Zhejiang, People's Republic of China
Correspondence: Qiaoding Dai Department of Rheumatology and Immunology, First Affiliated Hospital of Zhejiang Chinese Medical University, Hangzhou 310006, Zhejiang,

People's Republic of China

Tel/fax +86 57I 87072072

Email daiqiaoding@outlook.com
Purpose: Rheumatoid arthritis (RA) is a chronic, progressive autoimmune disease characterized by aggressive and symmetric polyarthritis. Mammalian target of rapamycin (mTOR) was reported to be a new target for RA therapy and its inhibitor rapamycin can significantly reduce the invasive force of fibroblast-like synoviocytes. Here, we determined the effect of curcumin to alleviate inflammation and synovial hyperplasia for the therapy of RA.

Materials and methods: Collagen-induced arthritis (CIA) was developed in Wistar rats and used as a model resembling RA in humans. Rats were treated with curcumin $(200 \mathrm{mg} / \mathrm{kg})$ and the mTOR inhibitor rapamycin $(2.5 \mathrm{mg} / \mathrm{kg})$ daily for 3 weeks. Effects of the treatment on local joint, peripheral blood, and synovial hyperplasia in the pathogenesis of CIA were analyzed.

Results: Curcumin and rapamycin significantly inhibited the redness and swelling of ankles and joints in RA rats. Curcumin inhibited the CIA-induced mTOR pathway and the RA-induced infiltration of inflammatory cells into the synovium. Curcumin and rapamycin treatment inhibited the increased levels of proinflammatory cytokines including IL-1 $\beta$, TNF- $\alpha$, MMP-1, and MMP-3 in CIA rats.

Conclusion: Our findings show that curcumin alleviates CIA-induced inflammation, synovial hyperplasia, and the other main features involved in the pathogenesis of CIA via the mTOR pathway. These results provide evidence for the anti-arthritic properties of curcumin and corroborate its potential use for the treatment of RA.

Keywords: rheumatoid arthritis, curcumin, rapamycin, mammalian target of rapamycin, collagen-induced arthritis

\section{Introduction}

Rheumatoid arthritis (RA) is a chronic, progressive autoimmune disease characterized by aggressive and symmetric polyarthritis. The incidence of RA is about $0.5 \%-1 \%$ in the population, and the annual incidence of new cases is about $5-50$ cases per $100,000 .{ }^{1,2}$ RA is not only a highly disabling disease, but can also cause lesions in organs and tissues outside the joints, such as the heart, lungs, and blood vessels. RA seriously affects the patient's quality of life and has a heavy burden on family and society. ${ }^{3,4}$ Currently, the pathogenesis of RA is unknown, and there is still no radical cure.

The basic lesion of RA consists of abnormal hyperplasia of the synovium. Fibroblast-like synoviocytes (FLS) produce many chemokines, proinflammatory factors, matrix metalloproteinases, and cathepsin to degrade the extracellular matrix and cartilage when in contact with immune cells that infiltrate into joints, or when exposed to growth factors and inflammatory cytokines such as TNF- $\alpha$ and IL-1. ${ }^{5}$ Therefore, the inhibition of abnormal hyperplasia, inflammatory cytokines, and matrix 
metalloproteinases in synovial cells is key to the treatment of RA synovitis.

Curcumin is a chemical composition extracted from the roots of Zingiberaceae and Araceae. Because curcumin has extensive pharmacological actions, a low rate of side effects is easily sourced and relatively cheap, and it has attracted great interest. ${ }^{6,7}$ Previous studies have shown that curcumin has defensive and therapeutic effects on the occurrence and development of RA. ${ }^{8-11}$

The mammalian target of rapamycin (mTOR) signaling pathway plays an important role in regulating cell growth, proliferation, differentiation, apoptosis, and the occurrence of tumors. Previous studies have shown that FLS with abnormal hyperplasia showed an aggressive characteristic similar to tumor cells. ${ }^{12}$ Rapamycin is an inhibitor of mTOR. Laragione and Gulko ${ }^{13}$ reported that the mTOR signaling pathway was involved in the regulation of FLS invasion, and that rapamycin significantly reduced the infiltration by FLS. Therefore, the mTOR signaling pathway might be a new target for RA therapy. In recent years, domestic and overseas studies have shown that curcumin exerts its inhibitory effect on tumor cell growth by targeting the mTOR signaling pathway as well as having a protective effect on the occurrence and development of arthritis. But whether curcumin can regulate RA by targeting the mTOR pathway is still unknown.

In this study, we analyzed whether curcumin modulates RA-induced inflammation and synovial hyperplasia and investigated the associated mechanism. We successfully established type II collagen-induced arthritis in rats and found that curcumin significantly increased the weight of rats and decreased the edema volume and arthritic score of rat hind paws. Curcumin also inhibited the mTOR pathway and the subsequent production of IL- $1 \beta$, TNF- $\alpha$, MMP-1, and MMP in the serum and synovium of RA rats.

\section{Materials and methods}

\section{Animals}

Overall, 40 male Wistar rats (aged 7-8 weeks) weighing $105 \pm 15$ g were purchased from Shanghai SLAC Laboratory Animal Co., Ltd., (Shanghai, People's Republic of China) and housed in an animal facility maintained at $25^{\circ} \mathrm{C}$ (humidity, 50\%-60\%) under a 12 hour light-dark cycle with free access to food and water. All experiments involving animals were approved and performed in accordance with the institutional guidelines for animal care of the animal ethics committee of Zhejiang Chinese Medical University.

\section{Reagents and antibodies}

Bovine type II collagen (CII; Chondrex, Inc., Redmond, WA, USA), curcumin (HY-N0005) and rapamycin (HY-10219) (MedChemExpress, Princeton, NJ, USA), were used in this study. Primary antibodies used in this study were all purchased from Cell Signaling Technology (Danvers, MA, USA) as follows: monoclonal anti-4E-BP1 antibody (9644), monoclonal anti-mTOR antibody (2983), monoclonal antip70 S6 kinase antibody (2708) and monoclonal anti-Akt1 antibody (75629).

\section{Arthritis model}

The rats were divided into two groups: control group ( $\mathrm{n}=10$, Con) and bovine type II collagen (CIA)-induced RA group $(n=30) \cdot{ }^{14,15}$ Bovine type II collagen was dissolved in $0.05 \mathrm{M}$ acetic acid to a final concentration of $2 \mathrm{mg} / \mathrm{mL}$ and vortexed overnight at $4{ }^{\circ} \mathrm{C}$. Subsequently, the bovine type II collagen acetic acid solution was emulsified with Freund's incomplete adjuvant at a ratio of 1:1 to a final concentration of $1 \mathrm{mg} / \mathrm{mL}$. Rats were immunized with the collagen emulsion by a subcutaneous booster injection into the tail at day 1 $(300 \mu \mathrm{L})$ and day $8(200 \mu \mathrm{L})$. The Con group was treated with normal saline.

\section{Arthritic scoring}

The severity of arthritis in each hind paw of CIA rats was scored and recorded as the mean score obtained by three experimental researchers. Severity was scored on a scale of $0-4$, where $0=$ no redness or swelling; $1=$ slight swelling in the ankle or redness in the foot; $2=$ progressive swelling, inflammation, and redness from the ankle to the midfoot; $3=$ swelling and inflammation of the entire foot; and $4=$ swelling and inflammation of the entire foot, with a loss of mobility. The arthritic index was calculated as a total score of $16 /$ animal (score of 4 for each limb). An arthritis index over 6 points can be judged to indicate the successful induction of the model.

\section{Treatment strategy}

Rats with the successful induction of RA were further divided into three groups: CIA-induced RA group ( $n=8$, CIA), rapamycin-treated CIA-induced RA group ( $n=8$, Rapamycin) and curcumin-treated CIA-induced RA group $(n=8$, Curcumin). At 21 days following the induction of RA via CIA, rats in the Rapamycin and Curcumin groups were gavaged with rapamycin $(2.5 \mathrm{mg} / \mathrm{kg})$ and curcumin $(200 \mathrm{mg} / \mathrm{kg})$ daily for 3 weeks. ${ }^{16}$ At 50 days following the induction of RA, the rats were sacrificed (Table 1). 
Table I Treatment regime in the CIA model

\begin{tabular}{l|l|l}
\hline Groups & Doses & $\begin{array}{l}\text { Route of } \\
\text { administration }\end{array}$ \\
\hline Con & - & - \\
CIA & - & - \\
Rapamycin & $2.5 \mathrm{mg} / \mathrm{kg}$ & Gavage \\
Curcumin & $200 \mathrm{mg} / \mathrm{kg}$ & Gavage \\
\hline
\end{tabular}

Abbreviations: $\mathrm{CIA}$, collagen-induced arthritis; Con, control.

\section{Histopathological analysis}

Histopathology analysis was performed on sections of paw joints. Briefly, paw joints were excised from the right hind limbs of rats after removal of the skin, superficial muscle, and tissue, followed by fixation in $4 \%$ polyoxymethylene solution at $4{ }^{\circ} \mathrm{C}$ for 1 week. The fixed specimens were placed in $14 \%$ EDTA solution (EDTA 500 g, hydrochloric acid $99.6 \mathrm{~mL}$, and deionized water $3.46 \mathrm{~L}$ ) for decalcification for 35 days at $4^{\circ} \mathrm{C}$, and the EDTA solution was changed every 5 days until the joint was soft. The joints were then dehydrated with alcohol, embedded in paraffin, sectioned into 4-6- $\mu \mathrm{m}$-thick slices, and placed on poly-L-lysine-coated slides. Then they were dewaxed in xylene, rehydrated through decreasing concentrations of ethanol, washed in PBS, and stained with H\&E.

\section{Immunohistochemical analysis}

Slides containing tissues were immersed in xylene followed by decreasing concentrations of alcohol $(100 \%, 90 \%$, and $70 \%$ ) and finally water. The slides were then dipped in PBS 3 times, incubated in $3 \%$ hydrogen peroxide for 10 minutes, and incubated in citrate buffer for 20 minutes at $95^{\circ} \mathrm{C}$. Blocking solution was poured onto the tissue sections and incubated for $30 \mathrm{~min}$ at $37^{\circ} \mathrm{C}$ and then incubated with primary antibody at $4{ }^{\circ} \mathrm{C}$ overnight followed by washing with PBS three times. The tissue sections were then incubated with secondary antibody at 1:200 dilution for $60 \mathrm{~min}$ and
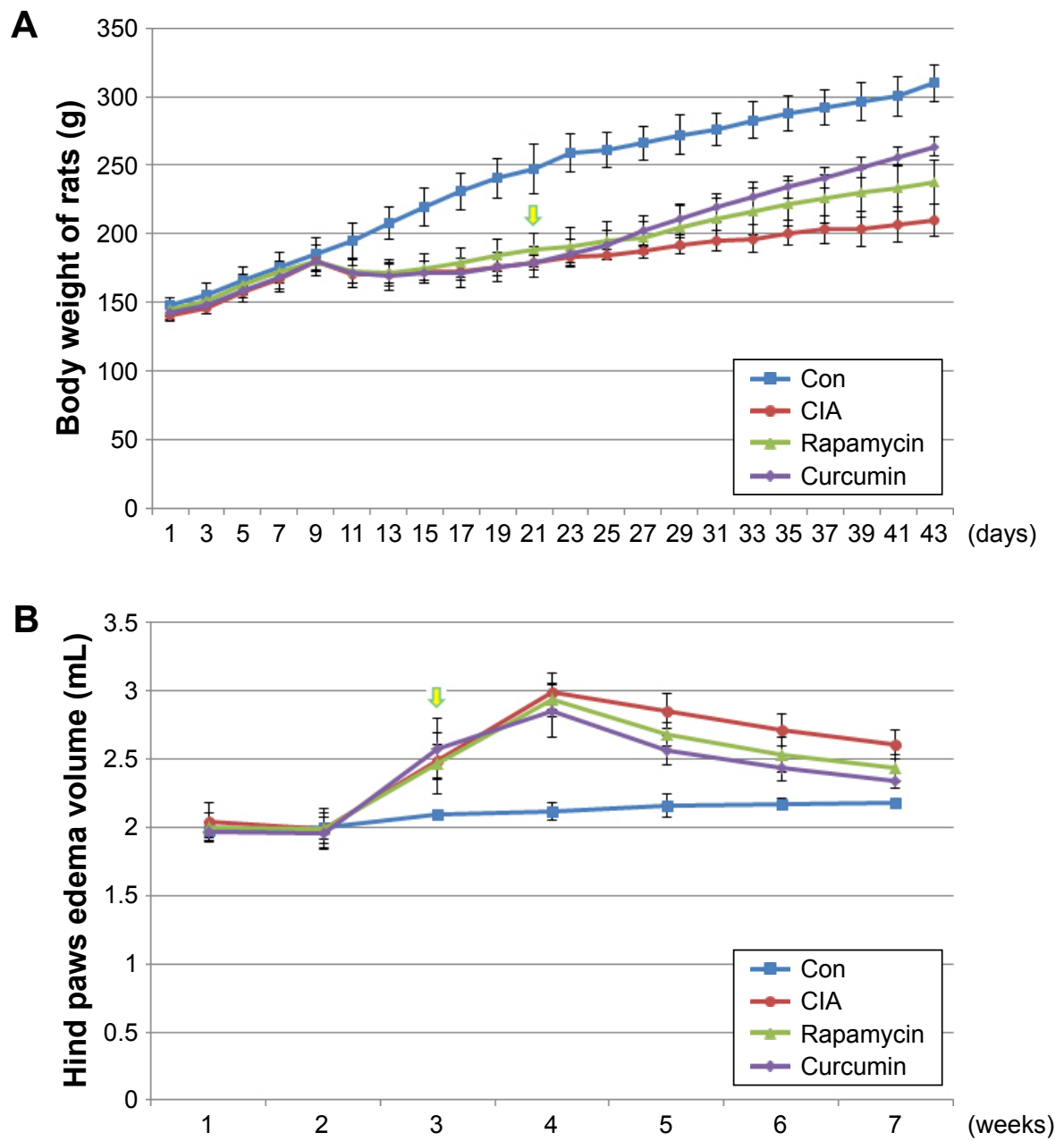

Figure I (Continued) 


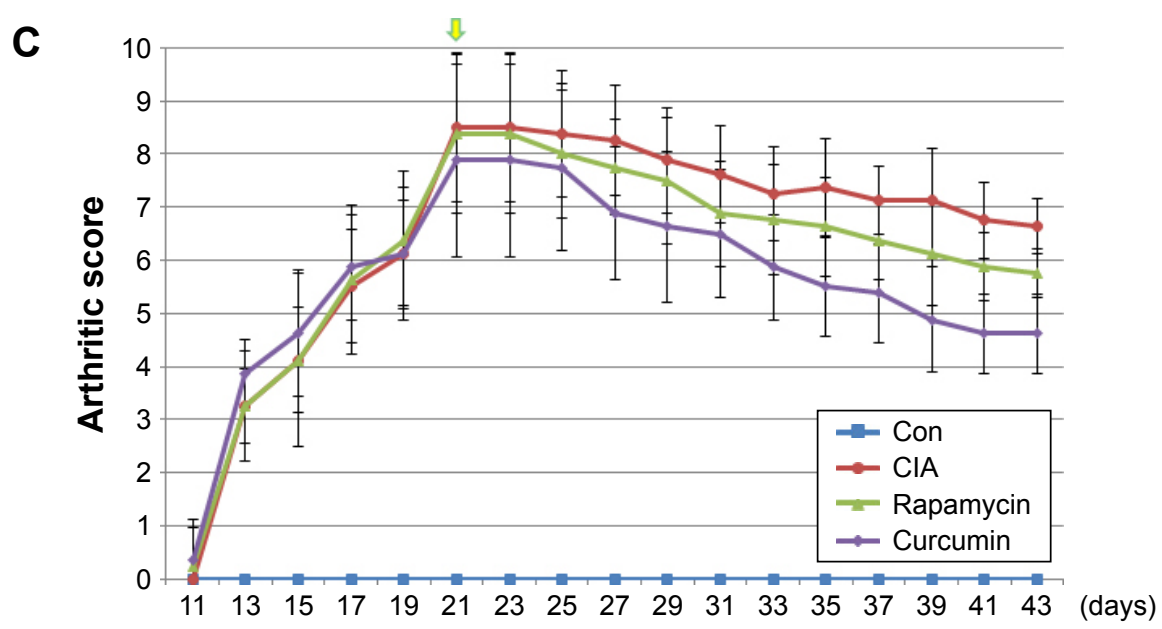

Figure I Collagen treatment successfully induced RA, and curcumin treatment alleviated CIA-related symptoms.

Notes: (A) The weight of rat treated with CIA, curcumin, and rapamycin. (B) The size of the full-toe in the Con, CIA, curcumin, and rapamycin rats. (C) The arthritic score of rat treated with $\mathrm{ClA}$, curcumin, and rapamycin rats.

Abbreviations: RA, rheumatoid arthritis; CIA, collagen-induced arthritis; Con, control.

washed with PBS at room temperature. Finally, tissue sections were stained with DAB and visualized under an optical microscope. Images were processed using Image-Pro Plus. The results were represented by the mean optical density of the positive reaction area. The data were compiled with the software package SPSS, version 19.0. Data were presented as the mean \pm standard error (SE). Statistical analysis of data was performed using the Student's $t$-test and rank sum test. A value of $P<0.05$ was considered statistically significant.

\section{Measurements of IL-I $\beta$, TNF- $\alpha$, and MMP-3 levels}

The levels of IL-1 $\beta$, TNF- $\alpha$, MMP-1, and MMP-3 in rat serum and synovium were determined by ELISA kits in accordance with the manufacturer's instructions.

\section{Statistical analysis}

The data were compiled with the software package SPSS, version 19.0. Data were presented as the mean \pm SE. Statistical analysis of data was performed using the Student's $t$-test and rank sum test. A value of $P<0.05$ was considered statistically significant.

\section{Results}

Collagen treatment successfully induces RA, and curcumin treatment alleviates

\section{CIA-related symptoms}

Rats were treated with bovine type II collagen (CIA) to induce RA. The weight of rats treated with CIA were decreased at day 9 and lasted for almost 10 days compared with the Con group (Figure 1A). Rats in all the treatment groups showed no signs of arthritis until day 10 . On day 14, all CIA rats had different degrees of redness and swelling in their hind paws, and their activity was mildly limited. The anterior foot joints of some rats also showed redness and swelling. On day 21 , the degree of redness reached its peak. The size
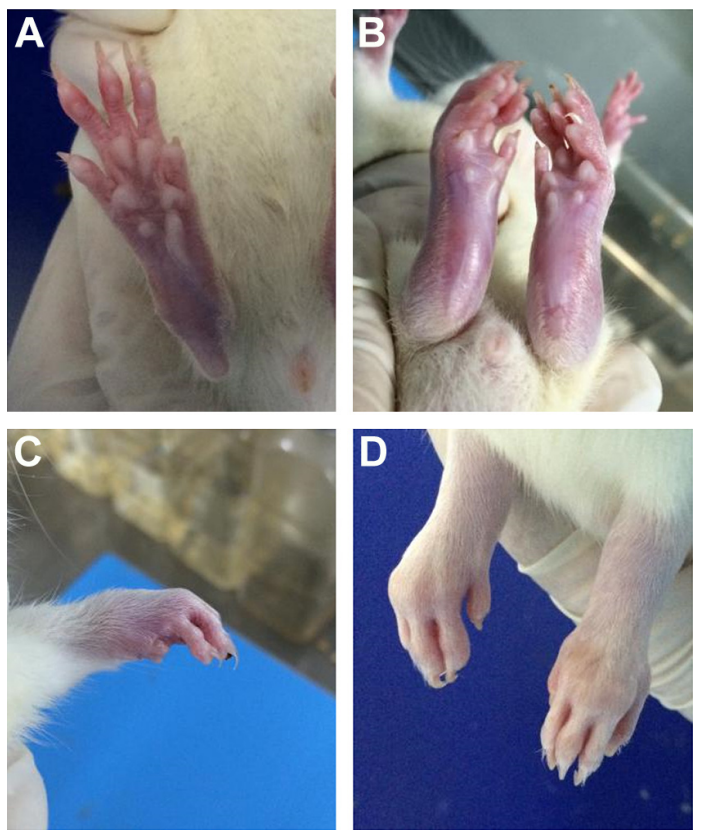

Figure 2 The foot joints of the $\mathrm{ClA}$ rats.

Notes: (A) Normal rat's hind feet. (B) Redness of the posterior foot joint. (C) Redness of the anterior foot joint. (D) Deformity of the hind foot joint. Abbreviation: $\mathrm{CIA}$, collagen-induced arthritis. 
A

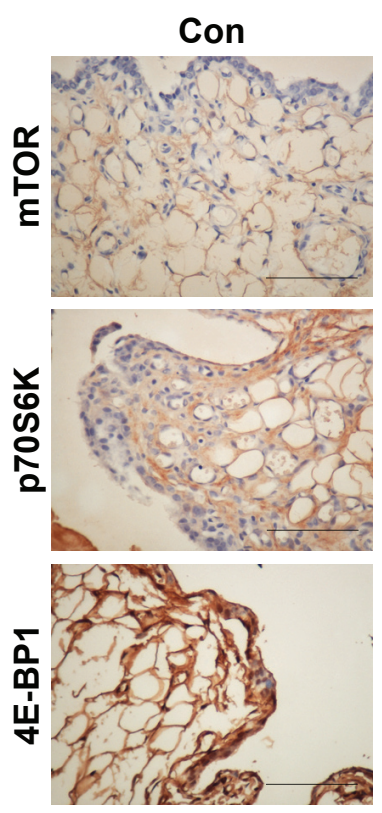

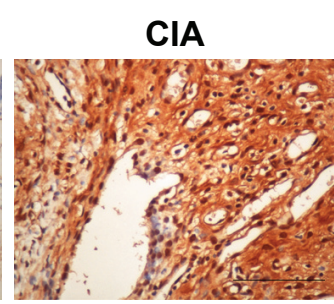
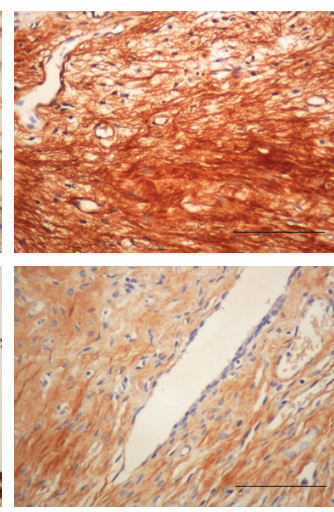

B
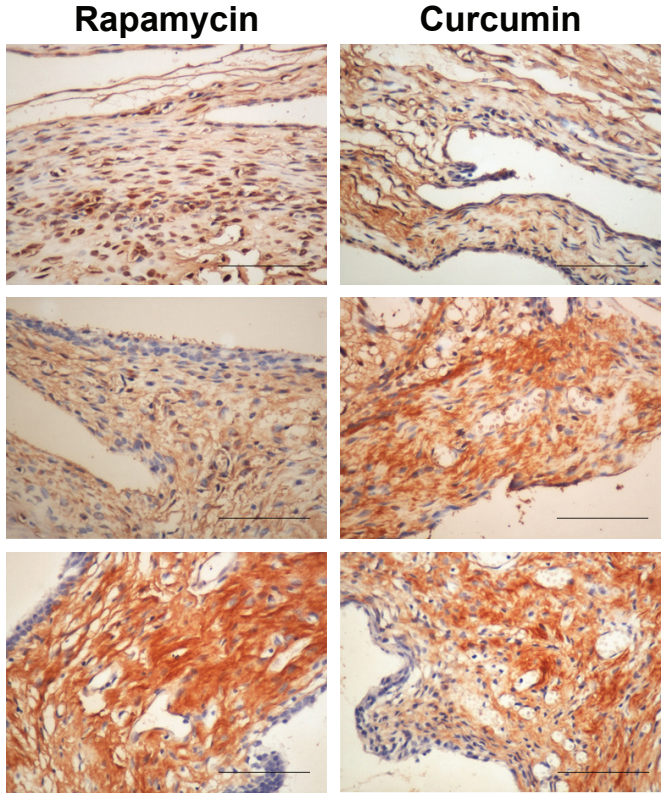

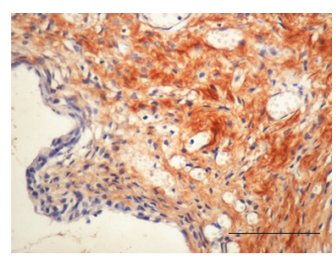

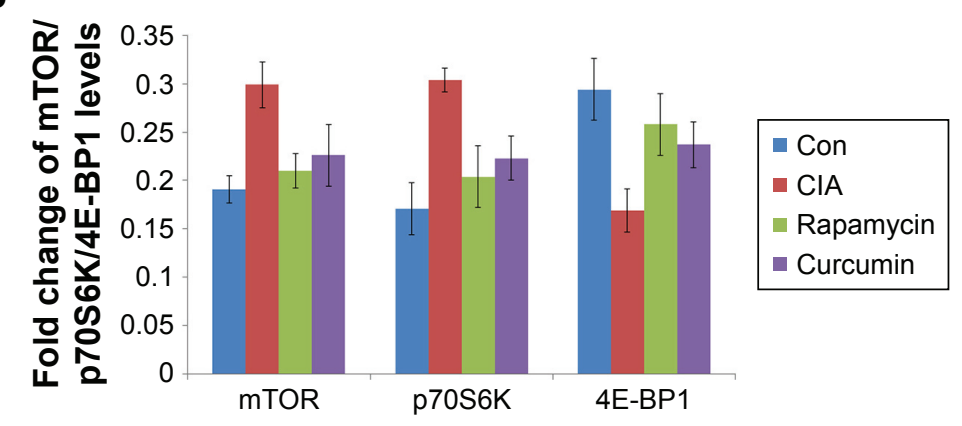

Figure 3 The expression of mTOR pathway in synovium.

Notes: $($ A, B $)$ The results of the immunohistochemistry of the synovium clearly indicated the expression of $\mathrm{mTOR}$, p70S6K, and 4E-BPI. Scale bar $=100 \mathrm{~mm}$. Abbreviations: CIA, collagen-induced arthritis; mTOR, mammalian target of rapamycin; Con, control.

of the full toe in the CIA group was significantly larger than that in the Con group (Figures 1B and 2), and activity was limited, as determined by dragging of the trailing foot. RA rats had increased arthritic scores from day 13 and this was significantly different compared with the Con group from day 13 onward (Figure 1C). At 21 days following the induction of RA, rats in the Curcumin groups were gavaged with curcumin $(200 \mathrm{mg} / \mathrm{kg})$ daily for 3 weeks. The weight of rats in the Curcumin group were increased compared with the CIA group (Figure 1A). The hind paw edema volume and arthritic scores were both decreased in curcumin-treated rats (Figure 1B and C).

\section{Curcumin inhibits the CIA-induced mTOR pathway in rat synovium}

Immunohistochemistry analysis of the synovium clearly indicated that the expression of mTOR was increased in the
CIA group compared with the Con group. Direct downstream substrates, p70S6 kinase (p70S6K) and eukaryotic initiation factor $4 \mathrm{E}$ binding protein 1 (4E-BP1) of the mTOR pathway were increased and decreased, respectively. Curcumin treatment decreased the CIA-induced level of mTOR and p70S6K and increased the 4E-BP1 level (Figure 3 and Table 2).

Table 2 The expression of mTOR, p70S6K, and 4E-BPI in rat synovium

\begin{tabular}{l|l|l|l}
\hline Groups & mTOR & p70S6K & 4E-BPI \\
\hline Con & $0.191 \pm 0.014$ & $0.17 I \pm 0.027$ & $0.294 \pm 0.032$ \\
CIA & $0.299 \pm 0.024 *$ & $0.304 \pm 0.012^{*}$ & $0.169 \pm 0.022^{*}$ \\
Rapamycin & $0.210 \pm 0.018^{*, * *}$ & $0.204 \pm 0.032^{*}, * *$ & $0.258 \pm 0.032^{*, * *}$ \\
Curcumin & $0.226 \pm 0.032^{*, * *, \#}$ & $0.223 \pm 0.023 * * *, \#$ & $0.237 \pm 0.024 *, *, \#$ \\
\hline
\end{tabular}

Notes: Data are presented as mean \pm SD. Significant effect of treatment compared to Con group, $* P<0.05$; compared to CIA group, $* * P<0.05$; compared to Rapamycin group, ${ }^{*}<<0.05$.

Abbreviations: CIA, collagen-induced arthritis; mTOR, mammalian target of rapamycin; Con, control. 
To identify the function of curcumin in the inhibition of the CIA-induced mTOR pathway, we used rapamycin $(2.5 \mathrm{mg} / \mathrm{kg})$, an mTOR inhibitor, to treat CIA rats. Similar to curcumin treatment, levels of mTOR and p70S6K in the Rapamycin group were decreased and 4E-BP1 was increased compared with the CIA group (Figure 3). The weight of rats in the Rapamycin group were increased compared with the CIA group, and the hind paw edema volume and arthritic scores were decreased similar to that in curcumin-treated rats (Figure 1).

\section{Curcumin inhibits the RA-induced infiltration of inflammatory cells into the synovium}

Assessment of the synovium histology showed disrupted synovial cell layers, inflammatory cell infiltration, and
A
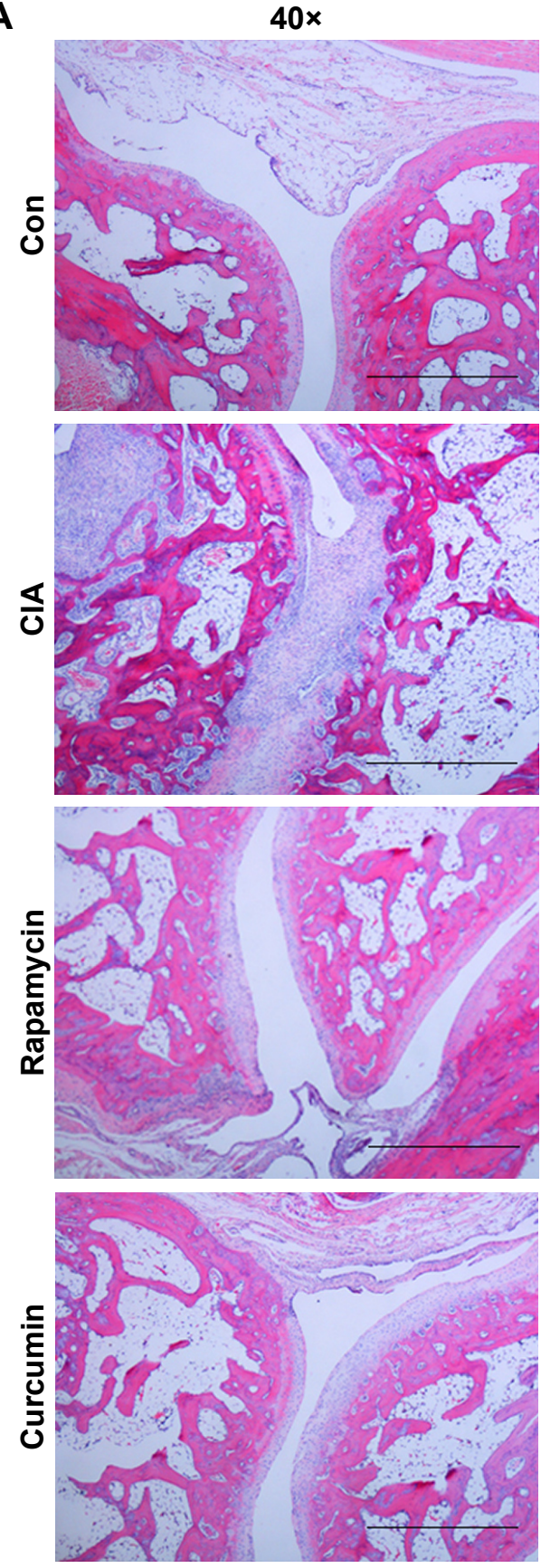

$400 x$
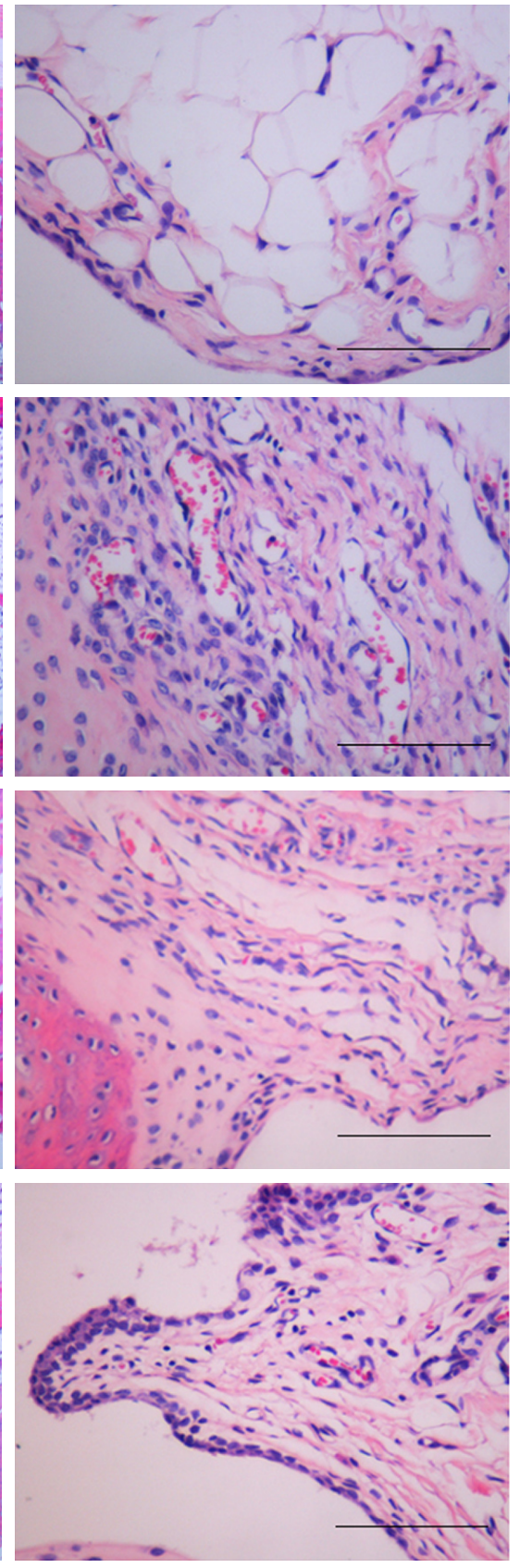

Figure 4 (Continued) 


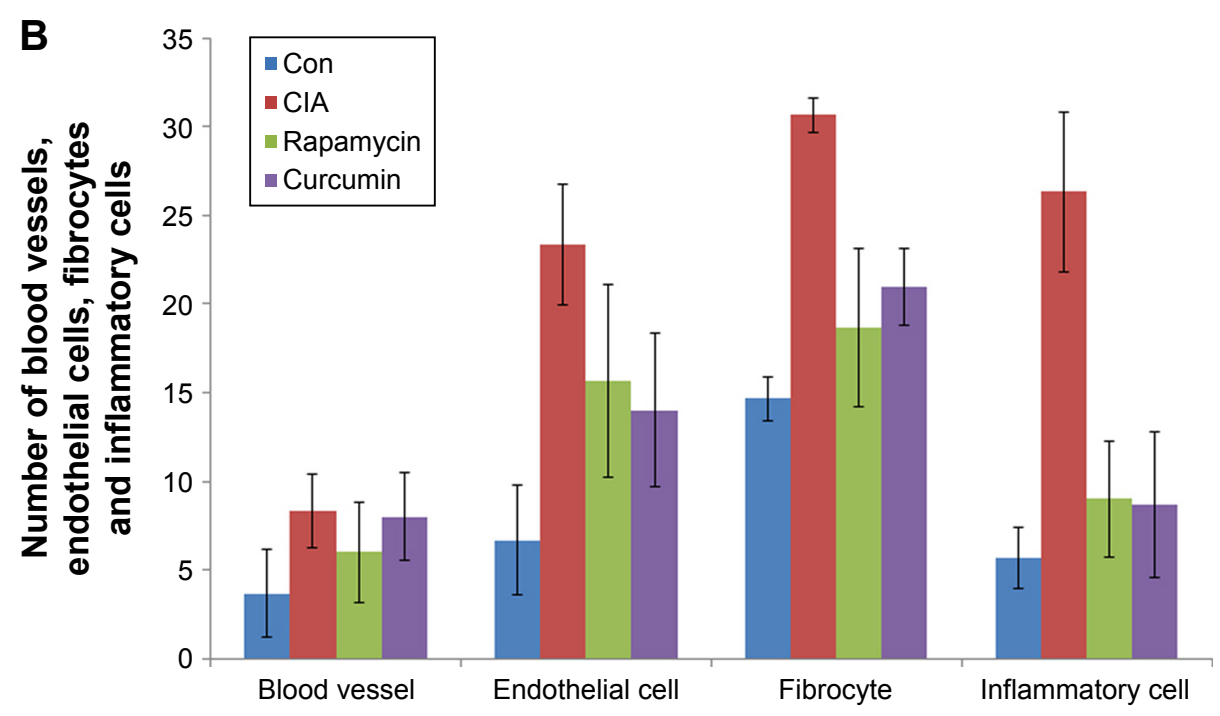

Figure 4 Curcumin inhibited RA-induced infiltration of inflammatory cells in synovium.

Notes: (A) The results of the histology in the synovial tissue of the Con, ClA, curcumin, and rapamycin groups. (B) The number of blood vessels, endothelial cells, fibrocytes, and inflammatory cells in the synovial tissue of Con, CIA, curcumin, and rapamycin groups.

Abbreviations: RA, rheumatoid arthritis; CIA, collagen-induced arthritis; Con, control.

synovial hyperplasia in the CIA group compared with the Con group, which showed an intact layer of synovial hyperplasia. Curcumin and rapamycin treatment efficiently alleviated CIA-induced inflammatory cell infiltration in the synovium as well as synovial hyperplasia (Figure 4).

\section{Curcumin alleviates proinflammatory cytokine expression in CIA rats}

To analyze the function of curcumin to treat RA rats, the levels of proinflammatory cytokines and proteinases, such as matrix metalloproteinases (MMPs) in rat serum and synovium were measured by ELISAs (Tables 3-6). IL-1 $\beta$, TNF- $\alpha$, MMP-1, and MMP-3 were all increased in serum of the CIA group compared with the Con group. Curcumin and rapamycin treatment inhibited the increased levels of IL- $1 \beta$, TNF- $\alpha$, MMP-1, and MMP-3 (Figure 5), and this was similar in the synovium (Figure 6).

Table 3 The expression of IL-I $\beta$ and TNF- $\alpha$ in rat serum

\begin{tabular}{l|l|l}
\hline Groups & IL-I $\beta$ & TNF- $\alpha$ \\
\hline Con & $158.58 \pm 34.00$ & $167.97 \pm 32.38$ \\
CIA & $483.06 \pm 73.06 *$ & $426.31 \pm 51.01 *$ \\
Rapamycin & $324.54 \pm 64.02 *, * *$ & $260.68 \pm 51.73 *, * *$ \\
Curcumin & $254.02 \pm 55.90 *, *, \#$ & $248.37 \pm 44.42 *, * *, \#$ \\
\hline
\end{tabular}

Notes: Data are presented as mean $\pm \mathrm{SD}(\mathrm{pg} / \mathrm{mL})$. Significant effect of treatment compared to Con group, $* P<0.05$; compared to the CIA group, $* * P<0.05$; compared to the rapamycin group, ${ }^{\#} P<0.05$.

Abbreviations: $\mathrm{CIA}$, collagen-induced arthritis; Con, control.

\section{Curcumin inhibits CIA-induced Aktl in the synovium}

We measured the expression of Akt1 by immunohistochemistry and found that Akt1 was significantly increased in the CIA group compared with the Con group. Curcumin treatment inhibited the level of Akt1 (Figure 7 and Table 7). These results indicated that curcumin might alleviate RA-induced inflammation and synovial hyperplasia by targeting the Akt1mTOR pathway in rats.

\section{Discussion}

RA is characterized by chronic arthromeningitis, persistent systematic inflammation, and the production of autoantibodies, especially to rheumatoid factors and cyclic citrullinated peptides. RA may lead to intraarticular cartilage injury, joint dysfunction, cardiovascular and pulmonary complications, and disability. ${ }^{17}$ RA pathogenesis is thought

Table 4 The expression of MMP-I and MMP-3 in rat serum

\begin{tabular}{l|l|l}
\hline Groups & MMP-I & MMP-3 \\
\hline Con & $162.77 \pm 11.14$ & $75.24 \pm 5.50$ \\
CIA & $219.13 \pm 14.23 *$ & $99.12 \pm 4.37 *$ \\
Rapamycin & $198.46 \pm 12.28 *, * *$ & $90.06 \pm 4.30 *, * *$ \\
Curcumin & $182.26 \pm 15.40 *, * *, \#$ & $84.19 \pm 5.09 *, * *, \#$ \\
\hline
\end{tabular}

Notes: Data are presented as mean \pm SD $(\mu \mathrm{g} / \mathrm{mL})$. Significant effect of treatment compared to Con group, ${ }^{*} P<0.05$; compared to the CIA group, ${ }^{* * P}<0.05$; compared to the rapamycin group, ${ }^{*} P<0.05$.

Abbreviations: $\mathrm{CIA}$, collagen-induced arthritis; Con, control. 
Table 5 The expression of IL-I $\beta$, TNF- $\alpha$ and IL-6 in rat synovium

\begin{tabular}{l|l|l}
\hline Groups & IL-I $\beta$ & TNF- $\alpha$ \\
\hline Con & $188.99 \pm 40.44$ & $137.83 \pm 34.07$ \\
CIA & $519.21 \pm 86.40^{*}$ & $433.28 \pm 79.80^{*}$ \\
Rapamycin & $403.18 \pm 31.88^{* * * *}$ & $294.72 \pm 54.14^{* * * *}$ \\
\hline Curcumin & $315.44 \pm 71.28 *, * * \#$ & $226.49 \pm 46.04 *, * *, \#$ \\
\hline
\end{tabular}

Notes: Data are presented as mean \pm SD $(\mathrm{pg} / \mathrm{mL})$. Significant effect of treatment compared to Con group, $* P<0.05$; compared to CIA group, $* * P<0.05$; compared to rapamycin group, ${ }^{\#} P<0.05$.

Abbreviation: $\mathrm{CIA}$, collagen-induced arthritis.

to involve complex interactions between genes and the environment; $50 \%$ of the risk for the development of RA is attributable to genetic factors while smoking accounts for the primary environmental risk. Because many currently available drugs induce side effects, patient compliance is very poor. Although the use of biological agents for RA has increased in recent years, it is expensive, and this has greatly limited the application of biological agents for RA. ${ }^{18-21}$ In the USA, the annual direct medical cost of each patient is about $\$ 13,500$, and the indirect cost is between $\$ 1,000$ and $\$ 33,000 .^{22}$

Curcumin is a yellow hydrophobic polyphenol derived from the herb turmeric and is widely distributed and cheap in the People's Republic of China. ${ }^{23-26}$ Curcumin possesses various pharmacological activities against many chronic diseases, including cancer, ${ }^{27,28}$ type II diabetes, ${ }^{29,30}$ multiple sclerosis, ${ }^{31,32}$ Alzheimer's disease, ${ }^{33}$ and atherosclerosis. ${ }^{34,35}$ However, studies on curcumin in RA are rare. Here, we found that curcumin effectively inhibited RA in a rat CIA model.

The pathogenesis of RA includes the following steps: synovial microvascular endothelial cells are activated, and the levels of surface adhesion molecules and chemokines are increased to promote leukocyte migration. Then, local hypoxia and cytokine induction induce angiogenesis and lymphangitic dysfunction forming the early characteristics of synovitis. These changes in the microenvironment, as well as synovial structure recombination and local fibroblast activation, contribute to the accumulation of inflammation in

Table 6 The expression of MMP-I and MMP-3 in rat synovium

\begin{tabular}{l|l|l}
\hline Groups & MMP-I & MMP-3 \\
\hline Con & $83.35 \pm 11.97$ & $32.96 \pm 4.96$ \\
CIA & $150.92 \pm 18.16^{*}$ & $66.37 \pm 5.32^{*}$ \\
Rapamycin & $115.70 \pm 11.57^{*, * *}$ & $49.07 \pm 6.57 *, * *$ \\
Curcumin & $101.16 \pm 13.67 *, *, \#$ & $42.46 \pm 4.41 *, * *, \#$ \\
\hline
\end{tabular}

Notes: Data are presented as mean $\pm S D(\mu \mathrm{g} / \mathrm{mL})$. Significant effect of treatment compared to the Con group, $* P<0.05$; compared to the CIA group, $* * P<0.05$; compared to the rapamycin group, $\# P<0.05$.

Abbreviations: CIA, collagen-induced arthritis; Con, control. the tissues of rheumatoid arthritis patients. ${ }^{1,17,36}$ At present, the full pathogenesis of RA is unclear.

The mTOR signaling pathway plays key roles in many physiological and pathological processes, including growth, proliferation, angiogenesis, metabolism, and autophagy. ${ }^{37-43}$ Feng and $\mathrm{Qiu}^{44}$ demonstrated that AKT inhibited chondrocyte proliferation and accelerated apoptosis and autophagy in RA rats through the $\mathrm{PI} 3 \mathrm{~K} / \mathrm{AKT} / \mathrm{mTOR}$ signaling pathway. Gambogic acid suppressed inflammation in RA rats via the $\mathrm{PI} 3 \mathrm{~K} / \mathrm{Akt} / \mathrm{mTOR}$ signaling pathway. ${ }^{45}$ But whether curcumin regulates RA by targeting the mTOR pathway is unknown. Our findings in this study showed that curcumin inhibited the expression of the mTOR pathway in CIA rats.

In RA, the dysfunction of FLS leads to hyperplastic synovium. The abnormal proliferation of FLS results from a loss of contact inhibition that plays a critical role in RA by producing inflammatory cytokines and proteinases, such as MMPs. Kim et $\mathrm{al}^{46}$ reported that the levels of $\mathrm{TNF} \alpha$ and IL-1 $\beta$ were elevated in the synovial fluid of RA patients and facilitated mucosal-associated invariant T (MAIT) cell migration. Zhai et $\mathrm{al}^{47}$ showed that paeonol, a natural product, had effects on inflammatory responses in IL-1 $\beta$-induced human fibroblast-like synoviocytes and rheumatoid arthritis progression by modulating the NF- $\kappa \mathrm{B}$ pathway. Zhou et $\mathrm{al}^{48}$ investigated the reliability and validity of serum MMP-3 levels and articular ultrasound scores to assess disease activity and therapeutic responses in RA patients and found that serum MMP-3 and US7 scores effectively reflected disease activity and therapeutic responses in patients with moderate-to-severe RA. In this study, we found that the levels of IL-1 $\beta$, TNF- $\alpha$, MMP-1, and MMP-3 were all increased in the CIA group in rat serum and synovium compared with the Con group. Furthermore, curcumin and rapamycin treatment both inhibited these increased levels of IL- $1 \beta$, TNF- $\alpha$, MMP-1, and MMP-3.

\section{Conclusion}

Curcumin inhibited inflammation, synovial hyperplasia, and the other main features of CIA-induced RA in rats via the mTOR pathway. Curcumin and rapamycin treatment decreased the CIA-induced levels of mTOR and p70S6K and increased 4E-BP1 levels. Moreover, the expression of Akt1 was inhibited in the curcumin group. Curcumin and rapamycin treatment inhibited the increased levels of IL- $1 \beta$, TNF- $\alpha$, MMP-1, and MMP-3. This provides evidence for the anti-arthritic properties of curcumin and corroborates its potential use for the treatment of RA. 
A

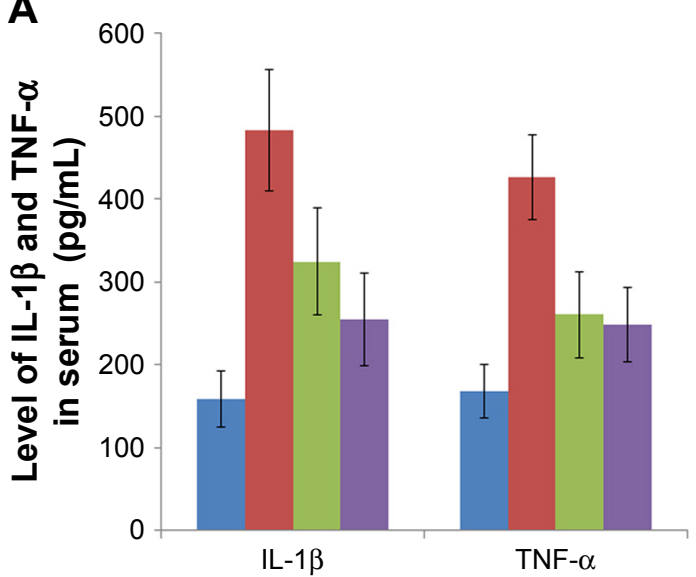

B

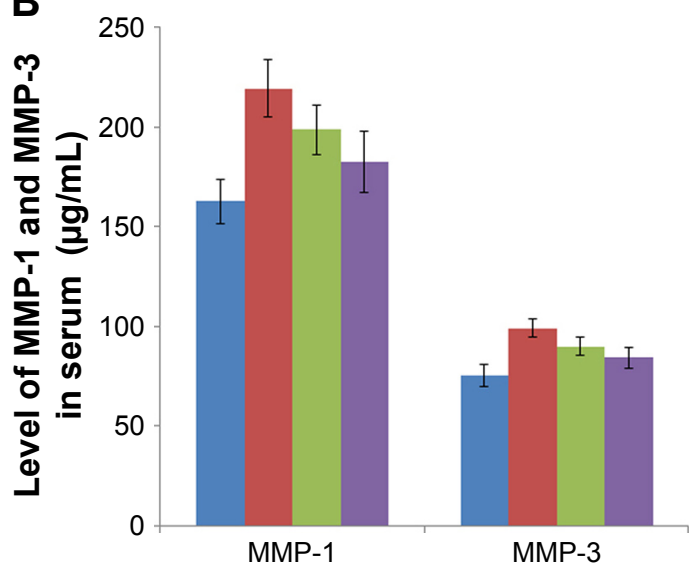

- Con $\quad$ ClA $\quad$ Rapamycin $\quad$ Curcumin

Figure 5 Curcumin alleviated proinflammatory cytokine expression in CIA rats' serum.

Notes: (A) The expression of IL-I $\beta$ and TNF- $\alpha$ in rats' serum. (B) The expression of MMP-I and MMP-3 in rats' serum.

Abbreviations: $\mathrm{CIA}$, collagen-induced arthritis; Con, control.

A

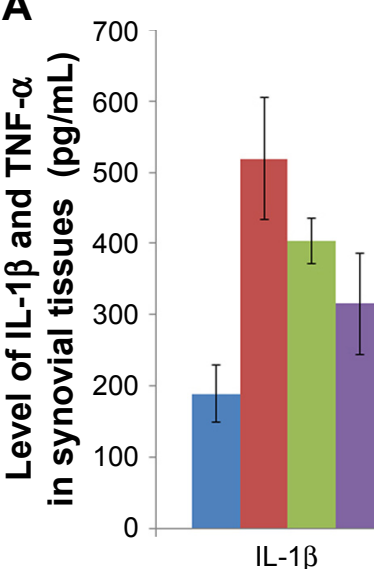

B

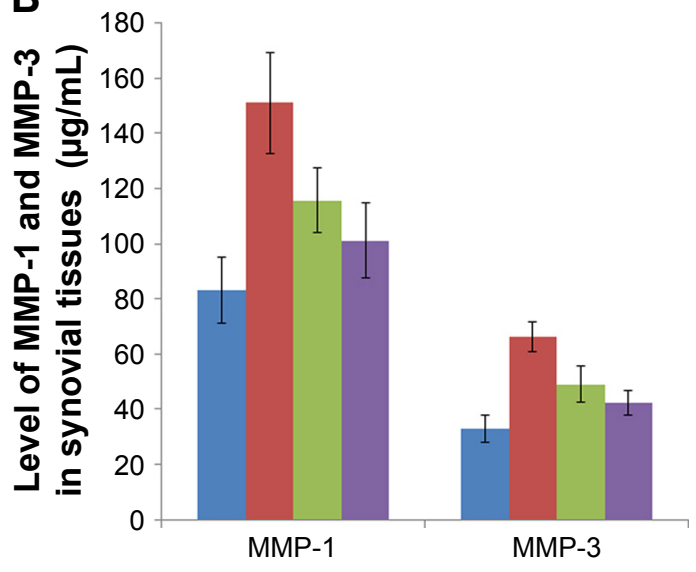

- Con $\quad$ - ClA $\quad$ Rapamycin $\quad$ Curcumin

Figure 6 Curcumin inhibition induces proinflammatory cytokine expression in CIA rats' synovium.

Notes: (A) The expression of IL-I $\beta$ and TNF- $\alpha$ in rats' synovium. (B) The expression of MMP-I and MMP-3 in rats' synovium. Abbreviations: $\mathrm{CIA}$, collagen-induced arthritis; Con, control.

A

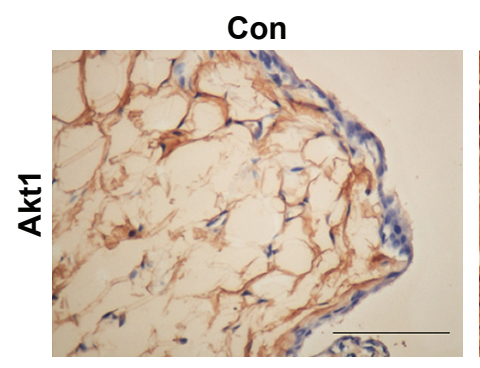

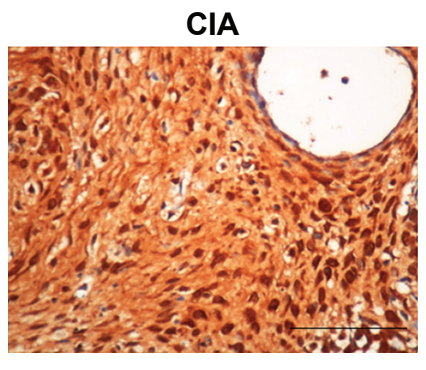

Curcumin

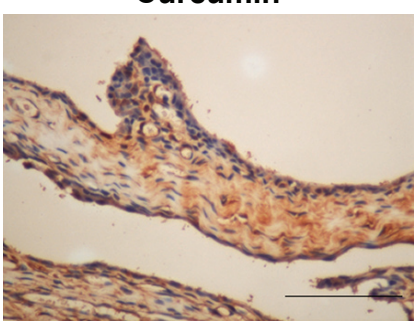

B

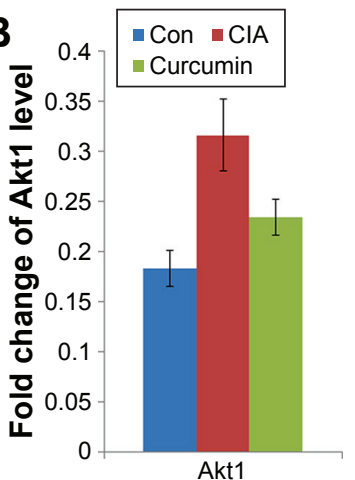

Figure 7 The expression of Aktl in synovium.

Note: (A and B) The results of the immunohistochemistry of the synovium clearly indicated the expression of Akt l.

Abbreviations: $\mathrm{CIA}$, collagen-induced arthritis; Con, control. 
Table 7 The expression of Aktl in rat synovium

\begin{tabular}{l|l}
\hline Groups & Akt I \\
\hline Con & $0.183 \pm 0.018$ \\
CIA & $0.316 \pm 0.036^{*}$ \\
Curcumin & $0.234 \pm 0.020^{* * * *}$ \\
\hline
\end{tabular}

Notes: Data are presented as mean \pm SD. Significant effect of treatment compared to Con group, $* P<0.05$; compared to the CIA group, $* * P<0.05$.

Abbreviations: $\mathrm{CIA}$, collagen-induced arthritis; Con, control.

\section{Acknowledgment}

This work was supported by the Medical Science and Technology Project of Zhejiang Province (grant number: 2014KYB166 and 2017KY503), the Traditional Chinese Medical Science and Technology Project of Zhejiang Province of China (Grant No. 2017ZB032 and 2014ZA028), the Educational Commission of Zhejiang Province of China (Grant No. Y201431280).

\section{Author contributions}

QDD, DZ, and LPX designed the study and interpreted the data. LPX and XWS helped in experiments and data collection. QDD and DZ performed statistical analysis and drafted the manuscript. All authors contributed to data analysis, drafting and revising the article, gave final approval of the version to be published, and agree to be accountable for all aspects of the work.

\section{Disclosure}

The authors report no conflicts of interest in this work.

\section{References}

1. Carmona L, Cross M, Williams B, Lassere M, March L. Rheumatoid arthritis. Best Pract Res Clin Rheumatol. 2010;24(6):733-745.

2. Hah JH, An SY, Sim S, et al. A population-based study on the association between rheumatoid arthritis and voice problems. Clin Rheumatol. 2016;35(7):1873-1878.

3. Scott DL, Wolfe F, Huizinga TW. Rheumatoid arthritis. Lancet. 2010; 376(9746):1094-1108.

4. Cross M, Smith E, Hoy D, Carmona L. The global burden of rheumatoid arthritis. Estimates from the global burden of disease 2010 study. Ann Rheum Dis. 2014;73(7):1316-1322.

5. Igarashi H, Yahagi A, Saika T, et al. A pro-inflammatory role for A20 and ABIN family proteins in human fibroblast-like synoviocytes in rheumatoid arthritis. Immunol Lett. 2012;141(2):246-253.

6. Subramani PA, Panati K, Narala VR. Curcumin nanotechnologies and its anticancer activity. Nutr Cancer. 2017;69(3):381-393.

7. Sundar Dhilip Kumar S, Houreld NN, Abrahamse H. Therapeutic potential and recent advances of curcumin in the treatment of aging-associated diseases. Molecules. 2018;23(4):E835.

8. Ahn JK, Kim S, Hwang J, et al. Metabolomic elucidation of the effects of curcumin on fibroblast-like synoviocytes in rheumatoid arthritis. PLoS One. 2015;10(12):e0145539.

9. Mande PP, Bachhav SS, Devarajan PV. Solid dispersion of curcumin as polymeric films for bioenhancement and improved therapy of rheumatoid arthritis. Pharm Res. 2016;33(8):1972-1987.
10. Shang W, Zhao LJ, Dong XL, et al. Curcumin inhibits osteoclastogenic potential in PBMCs from rheumatoid arthritis patients via the suppression of MAPK/RANK/c-Fos/NFATc1 signaling pathways. Mol Med Rep. 2016;14(4):3620-3626.

11. Amalraj A, Varma K, Jacob J, et al. A Novel highly bioavailable curcumin formulation improves symptoms and diagnostic indicators in rheumatoid arthritis patients: a randomized, double-blind, placebo-controlled, two-dose, three-arm, and parallel-group study. J Med Food. 2017; 20(10):1022-1030.

12. Firestein GS. Invasive fibroblast-like synoviocytes in rheumatoid arthritis. Passive responders or transformed aggressors? Arthr Rheum. 1996;39(11):1781-1790.

13. Laragione T, Gulko PS. mTOR regulates the invasive properties of synovial fibroblasts in rheumatoid arthritis. Mol Med. 2010;16(9-10): 352-358.

14. Tao Y, Wang Z, Wang L, et al. Downregulation of miR-106b attenuates inflammatory responses and joint damage in collagen-induced arthritis. Rheumatology. 2017;56(10):1804-1813.

15. Jiang $\mathrm{H}, \mathrm{Hu} \mathrm{H}$, Zhang $\mathrm{Y}$, et al. Amelioration of collagen-induced arthritis using antigen-loaded dendritic cells modified with NF- $\kappa \mathrm{B}$ decoy oligodeoxynucleotides. Drug Des Devel Ther. 2017;11:2997-3007.

16. Sabino V, Narayan AR, Zeric T, Steardo L, Cottone P. mTOR activation is required for the anti-alcohol effect of ketamine, but not memantine, in alcohol-preferring rats. Behav Brain Res. 2013;247:9-16.

17. Guo Q, Wang Y, Xu D, Nossent J, Pavlos NJ, Xu J. Rheumatoid arthritis: pathological mechanisms and modern pharmacologic therapies. Bone Res. 2018;6:15.

18. Mok CC. Morning stiffness in elderly patients with rheumatoid arthritis: what is known about the effect of biological and targeted agents? Drugs Aging. 2018;35(6):477-483.

19. Takanashi S, Nakazato T, Aisa Y, et al. The prognostic value of positron emission tomography/computed tomography in rheumatoid arthritis patients with methotrexate-associated lymphoproliferative disorders. Ann Hematol. 2018;97(9):1611-1618.

20. Pirmardvand Chegini S, Varshosaz J. Recent approaches for targeted drug delivery in rheumatoid arthritis diagnosis and treatment. ArtifCells Nanomed Biotechnol. Epub 2018 Apr 16.

21. Martín-López M, Carmona L, Balsa A, et al. Serum drug levels of biologic agents in the management of rheumatoid arthritis and spondyloarthritis: a systematic review. Rheumatol Int. 2018;38(6):975-983.

22. Kvien TK. Epidemiology and burden of illness of rheumatoid arthritis. Pharmacoeconomics. 2004;22(2 Suppl 1):1-12.

23. Huang Y, Cao S, Zhang Q, et al. Biological and pharmacological effects of hexahydrocurcumin, a metabolite of curcumin. Arch Biochem Biophys. 2018;646:31-37.

24. Hewlings SJ, Kalman DS. Curcumin: a review of its effects on human health. Foods. 2017;6(10):E92:92.

25. Shehzad A, Qureshi M, Anwar MN, Lee YS. Multifunctional curcumin mediate multitherapeutic effects. J Food Sci. 2017;82(9):2006-2015.

26. Rahmani AH, Alsahli MA, Aly SM, Khan MA, Aldebasi YH. Role of curcumin in disease prevention and treatment. Adv Biomed Res. 2018;7:38.

27. Murray-Stewart T, Casero RA. Regulation of polyamine metabolism by curcumin for cancer prevention and therapy. Med Sci. 2017; 5(4):E38:38.

28. Hamzehzadeh L, Atkin SL. The versatile role of curcumin in cancer prevention and treatment. A focus on PI3K/AKT pathway. J Cell Physiol. 2018;233(10):6530-6537.

29. Su LQ, Wang YD, Chi HY. Effect of curcumin on glucose and lipid metabolism, FFAs and TNF- $\alpha$ in serum of type 2 diabetes mellitus rat models. Saudi J Biol Sci. 2017;24(8):1776-1780.

30. Kim BH, Lee ES, Choi R, et al. Protective effects of curcumin on renal oxidative stress and lipid metabolism in a rat model of type 2 diabetic nephropathy. Yonsei Med J. 2016;57(3):664-673.

31. Xie L, Li XK, Takahara S. Curcumin has bright prospects for the treatment of multiple sclerosis. Int Immunopharmacol. 2011;11(3):323-330. 
32. Qureshi M, Al-Suhaimi EA, Wahid F, Shehzad O, Shehzad A. Therapeutic potential of curcumin for multiple sclerosis. Neurol Sci. 2018; 39(2):207-214.

33. Chen M, du ZY, Zheng X, Li DL, Zhou RP, Zhang K. Use of curcumin in diagnosis, prevention, and treatment of Alzheimer's disease. Neural Regen Res. 2018;13(4):742-752.

34. Hasan ST, Zingg JM, Kwan P, Noble T, Smith D, Meydani M. Curcumin modulation of high fat diet-induced atherosclerosis and steatohepatosis in LDL receptor deficient mice. Atherosclerosis. 2014;232(1):40-51.

35. Zhang S, Zou J, Li P, Zheng X, Feng D. Curcumin protects against atherosclerosis in apolipoprotein E-knockout mice by inhibiting toll-like receptor 4 expression. J Agric Food Chem. 2018;66(2):449-456.

36. Calabresi E, Petrelli F, Bonifacio AF, Puxeddu I, Alunno A. One year in review 2018: pathogenesis of rheumatoid arthritis. Clin Exp Rheumatol. 2018;36(2):175-184.

37. Lee DF, Hung MC. All roads lead to mTOR: integrating inflammation and tumor angiogenesis. Cell Cycle. 2007;6(24):3011-3014.

38. Martelli AM, Buontempo F, Mccubrey JA. Drug discovery targeting the mTOR pathway. Clin Sci. 2018;132(5):543-568.

39. Wee P, Wang Z. Epidermal growth factor receptor cell proliferation signaling pathways. Cancers. 2017;9(5):E52.

40. Yang D, Jiang T, Liu J, et al. Hormone regulates endometrial function via cooperation of endoplasmic reticulum stress and mTOR-autophagy. 2018;233(9):6644-6659

41. Linke M, Fritsch SD, Sukhbaatar N, Hengstschläger M, Weichhart T mTORC1 and mTORC2 as regulators of cell metabolism in immunity. FEBS Lett. 2017;591(19):3089-3103.
42. Roohi A, Hojjat-Farsangi M. Recent advances in targeting mTOR signaling pathway using small molecule inhibitors. J Drug Target. 2017;25(3):189-201.

43. Tee A. The target of rapamycin and mechanisms of cell growth. Int $J$ Mol Sci. 2018;19(3):880.

44. Feng FB, Qiu HY. Effects of Artesunate on chondrocyte proliferation, apoptosis and autophagy through the PI3K/AKT/mTOR signaling pathway in rat models with rheumatoid arthritis. Biomed Pharmacother. 2018;102:1209-1220.

45. Wu X, Long L, Liu J, et al. Gambogic acid suppresses inflammation in rheumatoid arthritis rats via PI3K/Akt/mTOR signaling pathway. Mol Med Rep. 2017;16(5):7112-7118.

46. Kim M, Yoo SJ, Kang SW, Kwon J, Choi I, Lee CH. TNF $\alpha$ and IL-1 $\beta$ in the synovial fluid facilitate mucosal-associated invariant T (MAIT) cell migration. Cytokine. 2017;99:91-98.

47. Zhai K-F, Duan H, Luo L, et al. Protective effects of paeonol on inflammatory response in IL-1 $\beta$-induced human fibroblast-like synoviocytes and rheumatoid arthritis progression via modulating NF- $\kappa \mathrm{B}$ pathway. Inflammopharmacology. 2017;25(5):523-532.

48. Zhou L, Wang G, Liu X, Song J, Chen L, Xu H. Matrix metalloproteinase-3 and the 7-joint ultrasound score in the assessment of disease activity and therapeutic efficacy in patients with moderate to severe rheumatoid arthritis. Arthritis Res Ther. 2017;19(1):250.
Drug Design, Development and Therapy

\section{Publish your work in this journal}

Drug Design, Development and Therapy is an international, peerreviewed open-access journal that spans the spectrum of drug design and development through to clinical applications. Clinical outcomes, patient safety, and programs for the development and effective, safe, and sustained use of medicines are the features of the journal, which

\section{Dovepress}

has also been accepted for indexing on PubMed Central. The manuscript management system is completely online and includes a very quick and fair peer-review system, which is all easy to use. Visit http://www.dovepress.com/testimonials.php to read real quotes from published authors.

Submit your manuscript here: http://www.dovepress.com/drug-design-development-and-therapy-journal 